\title{
Clinical Complexity in Middle-Aged and Older Adults With Diabetes:
}

\author{
The Health and Retirement Study
}

Caroline S. Blaum, MD, MS ${ }^{\star}$, Christine T. Cigolle, MD, MPH ${ }^{\dagger}$, Cynthia Boyd, MD, MPH ${ }^{\ddagger}$, Jennifer L. Wolff, PhD§, Zhiyi Tian, MA, MS ${ }^{\Uparrow}$, Kenneth M. Langa, MD, PhD", and David R. Weir, $\mathbf{P h D}^{* *}$

"Division of Geriatrics, Department of Internal Medicine, University of Michigan and Veterans Administration Health Center, Ann Arbor, Michigan

tDepartment of Family Medicine Center, University of Michigan and Veterans Administration Health Center, Ann Arbor, Michigan

$\mp$ Center on Aging and Health, Division of Geriatric Medicine and Gerontology, Johns Hopkins University School of Medicine, Baltimore, MD

$\S$ Department of Health Policy and Management, Johns Hopkins, Bloomberg School of Public Health, Baltimore, MD

IRegenstrief Center for Healthcare Engineering, Purdue University, West Lafayette, Indiana

"Division of General Medicine, Department of Internal Medicine, University of Michigan, and Veterans Affairs Health Services Research and Development Center of Excellence, VAAAHCS, and Institute for Social Research, University of Michigan

${ }^{* *}$ Institute for Social Research, University of Michigan, Ann Arbor, Michigan

\section{Abstract}

Background-Some patients with diabetes may have health status characteristics that could make diabetes self-management (DSM) difficult and lead to inadequate glycemic control, or limit the benefit of some diabetes management interventions.

Objective-To investigate how many older and middle-aged adults with diabetes have such health status characteristics.

Design-Secondary data analysis of a nationally representative health interview survey, the Health and Retirement Study, and its diabetes mail-out survey.

Setting/Participants-Americans aged 51 and older with diabetes $(n=3506$ representing 13.6 million people); aged 56 and older in diabetes survey $(\mathrm{n}=1132$, representing 9.9 million).

Measurements—Number of adults with diabetes and $(a)$ relatively good health; $(b)$ health status that could make DSM difficult (eg, comorbidities, impaired instrumental activities of daily living; and $(c)$ characteristics like advanced dementia and activities of daily living dependency that could limit benefit of some diabetes management. Health and Retirement Study measures included demographics. Diabetes Survey included self-measured $\mathrm{HbA}_{1 \mathrm{c}}$.

Copyright ( 92010 by Lippincott Williams \& Wilkins

Reprints: Caroline S. Blaum, MD, MS, Department of Internal Medicine and Geriatrics, University of Michigan, Research Scientist, Ann Arbor VA GRECC, Institute of Gerontology, 914 NIB, 300 N. Ingalls St., Ann Arbor, MI 48109. cblaum@ umich.edu. 
Results-Nearly $22 \%$ of adults $\geq 51$ with diabetes (about 3 million people) have health characteristics that could make DSM difficult. Another 10\% (1.4 million) may receive limited benefit from some diabetes management. Mail-out respondents with health characteristics that could make DSM difficult had significantly higher mean $\mathrm{HbA}_{1 \mathrm{c}}$ compared with people with relatively good health $(7.6 \%$ vs. $7.3 \%, P<0.04$. $)$.

Conclusions-Some middle-aged as well as older adults with diabetes have health status characteristics that might make DSM difficult or of limited benefit. Current diabetes quality measures, including measures of glycemic control, may not reflect what is possible or optimal for all patient groups.

The prevalence and incidence of type 2 diabetes are rising alarmingly in the United States, ${ }^{1,2}$ leading to concerns about worsening population health status and ever rising health care costs. Policy makers, clinicians, and people with diabetes are increasingly focused on improving diabetes management, including achieving glycemic control, lipid and blood levels, and blood pressure targets, which have been shown in randomized controlled trials (RCT's) to decrease some diabetes complications. ${ }^{3-6}$ Although many of these efforts have focused on improving diabetes management performance of physicians and other providers, diabetes self management (DSM) by patients is also critically important to achieve diabetes care targets and decrease complications.

Type 2 diabetes patients are a heterogeneous group of different ages, ethnicity, and socioeconomic status, with different diabetes characteristics, and widely varying health status. ${ }^{7} 8$ Some are healthy and functional with few complications and comorbidities; others have complex health status with multiple complications, comorbidities, and disabilities. We theorized that some people with diabetes and complex health status have health challenges that could decrease their DSM capabilities, and that some are so impaired they may experience little benefit from some diabetes management.

The goal of our research was to investigate how many middle-aged and older adults with diabetes in the United States would have complex health status potentially associated with DSM difficulty or limited benefit of some diabetes management. We defined 3 health status groups based on the literature and clinical experience. First, we defined a Relatively Healthy Group, with fewer than 3 chronic diseases, no cognitive or significant visual impairment, and one or no instrumental activities of daily living (IADL) dependencies. People in this group are similar to those studied in RCT's. Second, we defined a group with health characteristics that could make DSM difficult, whose members have one or more of the following characteristics: 3 or more chronic diseases (in addition to diabetes), mild cognitive impairment, severe vision impairment, and/or 2 or more IADL dependencies. Recent RCT's, $\left(\right.$ ACCORD $^{9,10}$ and ADVANCE ${ }^{11,12}$ ) concerning diabetes management may include some of these people. Third, we defined a Limited Benefit Group, containing people with the poorest health status, considered to include one or more of the following: moderate to severe cognitive impairment, 2 or more ADL dependencies, and/or residence in a long-term nursing facility. Little evidence is available regarding appropriate outcomes or processes of diabetes management for this group, many of whom may have limited life expectancy.

We investigated associations of these clinical groups with age, demographics, and diabetes characteristics. We focused particularly on the DSM Difficulty Group, because these people still have risk for further diabetes complications and would benefit from evidence-based diabetes management. We studied associations with increasing age, because many older adults with diabetes have multiple comorbidities and other conditions that present a DSM burden ${ }^{13}$; with sociodemographic characteristics, because blacks and Hispanics, and people with lower education and income are at high risk for poor glycemic control and diabetes complications ${ }^{14,15}$; and with poorer glycemic control and more severe diabetes because 
these problems could increase glycemic management challenges for those with DSM difficulty.

Using the Health and Retirement Study (HRS), ${ }^{16}$ a nationally representative health interview survey, we estimated the prevalence of people with diabetes and these health status groups, and the age distribution and demographic characteristics of the groups. In a subset of the HRS sample, those with diabetes who responded to the 2003 HRS Diabetes Mail-out Survey, ${ }^{14}$ we investigated whether differences in health status are related to diabetes severity and glycemic control. We hypothesized that the complex health status associated with possible DSM difficulty would be more common among older than middle-aged adults and would be associated with socioeconomic challenges, poor glycemic control, and more severe diabetes.

\section{METHODS}

\section{Study Design}

This study is a cross-sectional secondary data analysis of a single wave of a nationallyrepresentative health interview panel survey, and includes a substudy using data from the previous year.

\section{Data and Participants}

These data are from the 2004 wave of the HRS and its supplemental 2003 Diabetes MailOut Survey. HRS wave 2004 is the wave closest in time to the 2003 Mail-Out Survey, which includes self-collected $\mathrm{HbA}_{1 \mathrm{c}}$ data for respondents. The HRS is a biennial longitudinal health interview survey of adults aged 51 and older in the United States. It is sponsored by the National Institute on Aging and fielded by the Institute for Social Research at the University of Michigan. The survey employs a multistage probability sample of households that is nationally representative. Nursing home residents are included. Sampling weights are available for each wave, allowing nationally-representative estimates to be made. When an eligible respondent was unable to be interviewed (eg, due to medical and/or cognitive problems), a proxy respondent was recruited to provide information ( $8.95 \%$ of interviews in 2004). Interviews were administered by phone (74.47\%) or in-person $(25.53 \%)$.

Of the 18,929 HRS respondents interviewed in 2004, representing 82 million adults 51 years and older in the United States in 2004, 3506 (18.5\%) reported being told by a doctor that they had diabetes. These respondents represented 13.6 million adults aged 51 and older with diabetes in the United States in that year. In 2004, the HRS sample was refreshed, adding people aged 51 to 56 to the nationally representative sample.

Data from the 2003 Diabetes Mail-Out Survey, which collected additional information on diabetes duration and management, were linked to 2004 HRS data. This supplement was sent to 2350 HRS respondents who reported diabetes in 2002; 1901 completed the survey (80.9\%). These respondents were from the 1998 cohort before ages 51 to 56 were refreshed in 2004 so only ages 56 and over are nationally representative. $\mathrm{HbA}_{1 \mathrm{c}}$ levels were obtained using a self-administered finger-stick kit to collect blood spot samples; 1233 successfully completed the $\mathrm{HbA}_{1 \mathrm{c}}$ blood spot samples (52.5\%). ${ }^{14}$ Black and Latino ethnicity, lower education and income levels, and longer duration of diabetes were associated with not returning the $\mathrm{HbA}_{1 \mathrm{c}}$ kits. Diabetes study weights correct for nonresponse using a propensity model and reweighted participants who completed the kit to be representative of all study participants. Of the 1901 respondents, 4 were excluded for missing data on race or ethnicity, 49 respondents under the age of 56 were excluded for sampling weights of zero, 649 respondents were excluded for lacking a valid $\mathrm{HbA}_{1 \mathrm{c}}$ score or having a sampling weight of 
zero for a nonage related reason, and 67 were excluded because they were not present in the 2004 wave, giving a final analytic sample of $1132(48 \%)$.

The HRS and the Diabetes Mail-Out Survey were approved by the Institutional Review Board at the University of Michigan. The data used for this analysis are publicly available and contain no unique identifiers.

\section{Description of Health Status in Diabetes}

On the basis of clinical insight and the literature, we defined 3 health status groups as detailed in the Introduction:

1. A Relatively Healthy Group: within this group, we further distinguished a very healthy group with either no comorbidities, or comorbidities constrained to osteoarthritis and hypertension, and with no impairments.

2. A group with health characteristics that could make DSM implementation difficult. This is the group of the most theoretical interest. A similar idea based on multiple comorbidities was suggested by Boyd et al. ${ }^{13}$ We extended this definition to include people with multiple comorbidities and/or any one of the following: mild cognitive impairment, poor vision, and 2 or more IADL impairments.

3. A Limited Benefit Group, containing people with the poorest health status, with one or more of the following: moderate to severe cognitive impairment, 2 or more ADL dependencies, and/or residence in a long-term nursing facility.

These groups are mutually exclusive and together include all respondents. We evaluated these clinical groups among HRS respondents with diabetes in 2004 by age group (51-64 [middle-age], 65-75, and over 75) and by demographics.

Diabetes Mail-Out respondents with available $\mathrm{HbA}_{1 \mathrm{c}}(\mathrm{n}=1132)$ were stratified into the 3 clinical groups. For this subset, we evaluated the associations of clinical group with: age group: mean, median, 25th and 75th percentile of $\mathrm{HbA}_{1 c}$; diabetes duration $\geq 10$ years, and insulin use.

\section{Variables and Their Measurement Chronic Diseases}

In the 2004 wave of the HRS, respondents reported whether or not a physician had ever diagnosed them with certain chronic diseases and answered questions about the activity/ severity of each (Available at: http://hrsonline.isr.umich.edu/docs/userg/dr-009.pdf):

- Hypertension: requiring medication.

- Heart disease: requiring medication.

- Chronic lung disease: requiring medication or other treatment.

- Cancer: cancers (excluding skin) diagnosed in 2002 to 2004.

- Musculoskeletal conditions: arthritis (unspecified type) requiring medication or other treatment and/or joint replacement in the previous 2 years and/or hip fracture in the previous 2 years.

- Stroke: requiring medication and/or having remaining problems.

- Psychiatric problems: requiring medication and/or therapy.

- We summed these diseases to derive a comorbidity count that was used to define the clinical groups. 


\section{Cognitive Impairment}

The HRS assesses cognitive impairment in 2 ways. For self-respondents, cognitive impairment is determined using a performance-based measure, a modified version of the Telephone Interview for Cognitive Status. ${ }^{17-19}$ We defined mild cognitive impairment as a score of 8 to 10 on the 35-point cognitive scale for those aged 65 and older and as a score of 5 on the 27-point cognitive scale for those 51 to 64 . We defined moderate/severe cognitive impairment as a score of 7 or below for those aged 65 and older and 4 or below for those 51 to 64 . These cut-points have been used in prior studies. ${ }^{20}$ Detailed information on the HRS cognitive measures is available on the HRS website (Available at: http://hrsonline.isr.umich.edu/docs/userg/dr-006.pdf).

For respondents unable to complete the interview, a combination of proxy respondent assessment and interviewer assessment of the respondent's memory was used to determine the presence and degree of cognitive impairment. ${ }^{17,18}$

\section{Disability}

Our analysis included dependency (having difficulty and receiving assistance) in 5 ADL's (bathing, dressing, eating, toileting, and transferring) and 5 IADL's (preparing meals, shopping, managing money, using the telephone, and managing medications).

\section{Vision Impairment}

Vision impairment was defined as poor eyesight despite the use of corrective lenses or blindness.

\section{Living Status}

Respondent living status included living in the community alone, living in the community with others (eg, family, friend, supported living), or living in a nursing facility for long-term custodial care.

\section{Demographic Characteristics}

Demographic variables included age (stratified into 3 age groups as described previously), gender, race (white, African-American, Hispanic), marital status (married, unmarried), educational attainment, and low net worth (total household assets minus current debt). ${ }^{16}$

\section{Diabetes Characteristics}

$\mathrm{HbA}_{1 \mathrm{c}}$, diabetes duration and insulin use are available only for the Diabetes Mail-Out Survey subgroup. The self-measured $\mathrm{HbA}_{1 \mathrm{c}}$ assay (At-Home; FlexSite Diagnostics Inc, Palm City, Florida) uses the Roche Unimate immunoassay and the Cobas integral analyzer (F. Hoffman-LaRoches Ltd., Basel, Switzerland) calibrated to a synthetic $\mathrm{HbA}_{1 \mathrm{c}}$ standard. This test has been evaluated against Diabetes Control and Complications Trail reference technology and extensively tested in the laboratory and company-sponsored supplements to clinical trials. The manufacturer reports a test coefficient of variation of $\leq 2.54 \%$, within the $<5 \%$ recommendation of the American Diabetes Association. ${ }^{21}$

\section{Statistical Analysis}

To adjust for the complex sample design of the HRS, the differential probability of selection, and nonresponse to the Diabetes Mail-Out Survey and $\mathrm{HbA}_{1 \mathrm{c}}$ testing, all analyses were appropriately weighted using the statistical package STATA (Release 9.0, Stata Corp, College Station, TX), producing national population estimates. ${ }^{22}$ Standard descriptive methods were used in estimating prevalence of respondents' demographic and clinical 
characteristics; in estimating the prevalence of respondents meeting criteria for clinical groups; in determining confidence intervals; and in making comparisons between groups. As described above, the diabetes mail-out survey yielded only a $48 \%$ useable sample for $\mathrm{HbA}_{1 \mathrm{c}}$. However, much information about nonrespondents is known because all those selected for the Diabetes Survey had responded to the HRS, so propensity model reweighting was done to achieve as nearly as possible a nationally representative sample. However $\mathrm{HbA}_{1 \mathrm{c}}$ was not available before the Diabetes Survey and therefore could not be included in these models.

\section{RESULTS}

Figure 1 illustrates the distribution of adults with diabetes in the different clinical groups by age. In this figure, the Relatively Healthy Group is subdivided into an Extremely Healthy Group. Over one-quarter of those aged 51 to 64 and nearly one-third of those 65 to 75 were in the DSM Difficulty or the Limited Benefit Groups, compared with about $47 \%$ of adults 76 and older. That means, however, 53\% of adults 76 and older (representing 1.6 million individuals) fell in the Relatively Healthy Group. In absolute numbers, there are more people in the 51 to 64 age group who are in the DSM Difficulty or Limited Benefit Groups than there are in Groups among older ages. As shown in Table 1, adults with diabetes in the 51 to 64 group comprised $41 \%$ of patients likely to have DSM difficulty and $31 \%$ of patients likely to have limited benefit. Population estimates and prevalences from the weights are rounded for ease of reading.

Table 1 compares the distribution of sociodemographic characteristics of people with diabetes aged 51 years and older in each of the clinical groups; most distributions were significantly different among groups. Those in the DSM Difficulty and the Limited Benefit Groups were more likely to be female, from an ethnic minority group, and unmarried; to have less education and net worth; and to live alone. (We included living in a long-term stay nursing facility as part of our definition of the Limited Benefit Group, discussed below.)

Tables 2 and 3 show results from the substudy of Diabetes Survey respondents $(n=1132)$ with $\mathrm{HbA}_{1 \mathrm{c}}$. Proportions for Table 2 are nationally representative for aggregate analyses. Table 2 describes the diabetes characteristics of people in the clinical groups. Table 3 stratifies the $\mathrm{HbA}_{1 \mathrm{c}}$ analyses by age group. Table 2 shows that those in the DSM Difficulty Group are significantly more liked to have higher mean $\mathrm{HbA}_{1 \mathrm{c}}$ values, diabetes of longer duration, and to be treated with insulin when compared with the Relatively Healthy Group. There was no significant difference between the Limited Benefit Group and Relatively Health Groups except for use of insulin. The median is different from the mean for all groups; the percentile analysis demonstrated that $25 \%$ of people in the DSM Difficulty Group and Limited Benefit Group had $\mathrm{HbA}_{1 \mathrm{c}}$ over 8.0\%. In Table 3, the $\mathrm{HbA}_{1 \mathrm{c}}$ means and medians within the Groups are stratified by age group. For the 56 to 64 and the 76 and older age groups, the mean $\mathrm{HbA}_{1 \mathrm{c}}$ level among people in the DSM Difficulty Group was significantly higher compared with the Relatively Healthy Group. Also, among the 56 to 64 year olds, the percentile analysis showed a wide distribution with $25 \%$ of people in this group having $\mathrm{HbA}_{1 \mathrm{c}}$ over $9.9 \%$.

\section{DISCUSSION}

Our study demonstrates that a substantial number of middle-aged and older adults with diabetes have health status challenges that could make DSM difficult. A smaller number are so impaired that some diabetes management interventions, such as glycemic control at usual targets, are likely to have limited benefit. Although the proportion of people over 75 with diabetes in these health status groups is higher, the absolute number is higher for people in 
the younger age groups. Thus, age is an inadequate criterion for determining which people with diabetes may need support in DSM or reevaluation of diabetes care interventions.

Although health status is more likely to be related to problems, and sometimes, advisability, of achieving current diabetes management targets than is age, policy related to diabetes can be based more on age than health status. For example, people over 75 are excluded from most diabetes quality measures and, until recently, from the RCT's that developed the evidence base for diabetes management. Our data demonstrate (Fig. 1), however, that 53\% of people with diabetes over 75 are relatively healthy and will probably have sufficient life expectancy to benefit from diabetes management, although the appropriate studies are not yet available.

Our clinical group of focus, the DSM Difficulty Group, has sociodemographic challenges including lower education, lower net worth and living alone. The characteristics of their diabetes also indicate higher prevalence of severe and poorly controlled disease, with significantly higher $\mathrm{HbA}_{1 \mathrm{c}}$, longer duration of diabetes and more insulin use when compared with relatively healthy people. Among this Group, those aged 56 to 64 had a substantially higher mean $\mathrm{HbA}_{1 \mathrm{c}}$ than those in the Relatively Healthy Group and, although we did not do statistical comparisons, than those with DSM Difficulty within the other age groups. Future research is needed to understand why clinical characteristics associated with DSM Difficulty impact this age group disproportionately. As the median $\mathrm{HbA}_{1 \mathrm{c}}$ demonstrates, some in this age group achieve glycemic targets, so future research should investigate group comparisons in more detail.

We cannot distinguish in this cross-sectional study whether increased hyperglycemia in the DSM Difficulty Group is due to the severity of their diabetes, their decreased DSM ability, or both. Regardless, these patients need to improve glycemic and other diabetes management targets in the face of broad-based challenges. However, it is precisely these people who should benefit from high quality diabetes management that offers the potential to prevent additional diabetes complications ${ }^{23}$; these patients are not excluded from measurement of provider diabetes care quality (unless they are over 75).

The Limited Benefit Group is small, and nonresponse to $\mathrm{HbA}_{1 \mathrm{c}}$ as well as small sample size limit information about this group. Also, there can be other definitions than the one we used to define this group; we proposed a clinical definition rather than life expectancy because clinical characteristics are what physicians see, and some of these people live for some time. We proposed this group because it a vulnerable, high cost group, heterogeneous in age, and it is not known whether these highly impaired people experience additional risks from diabetes that can be mitigated by treatment. Our data suggest $\mathrm{HbA}_{1 \mathrm{c}}$ in this group is not significantly different from the Relatively Healthy Group; this may be due to small sample size and nonresponse, or because many of those in this group would have caregivers. Research using later waves of the HRS is needed to investigate the health outcomes and mortality experience of this group.

A chief strength of this study is that it is based on a large, nationally representative survey that provides data on people with a wide range of ages and health status, contains information on cognitive performance, ADLs, and IADLs and is linked to the Diabetes Mail-Out Survey with finger-stick $\mathrm{HbA}_{1 \mathrm{c}}$ levels. Perhaps most importantly, the HRS is a biennial longitudinal survey. Therefore, our study can be the basis of future longitudinal studies to identify health trajectories of the health status groups, and to examine the association of the groups with mortality and other health outcomes.

The study has several limitations. The HRS is based on self-report data for chronic diseases, and is limited by the questions included in the survey. The Diabetes Mail-out Survey had 
only a $48 \%$ usable response rate for $\mathrm{HbA}_{1 c}$; the implications of this have been studied in published research. ${ }^{14}$ Other than in aggregate analyses, some findings from the Diabetes Survey cannot be considered nationally representative and the sample sizes are small for some analyses. However, in 2006 and $2008, \mathrm{HbA}_{1 \mathrm{c}}$ is again being measured in half the sample each year with improved response rate. These data will soon be available for comparison. The $\mathrm{HbA}_{1 \mathrm{c}}$ level was the only measure of quality investigated in this research but future studies will use newly available Medicare claims (up to 2005 only) and blood pressure measures (years 2006 and 2008) to study a wider range of diabetes management interventions and intermediate outcomes.

Our study demonstrates that there is a substantial number of middle-aged and older adults with diabetes in the United States, who have clinically complex health status with multiple social and diabetes risks, and a smaller number who are very highly impaired. These findings demonstrate the complexity of performance-based reimbursement for providers. There are several important and large patient groups for whom we have insufficient information and care delivery mechanisms to assure high quality diabetes care: people 75 and older, many of whom might benefit from high quality diabetes management; patients with DSM difficulty who need high quality care and sometimes additional assistance, but might also make the physician "look bad" and receive less reimbursement under performance-based reimbursement; and over a million highly vulnerable people who may receive limited benefit from some diabetes management but for whom we have little information about what constitutes diabetes care quality.

Current strategies to improve diabetes management and outcomes usually focus on physician performance. However, people with diabetes ultimately must manage their disease and achieve clinical goals. For the surprising number of middle-aged as well as older diabetes patients who face DSM challenges, supporting their self-management efforts by interventions such as patient and caregiver education, and organizing home medical and personal care is a substantial challenge for primary care providers. Improved diabetes quality measures could include prioritization of management interventions ${ }^{24}$ and incentives for care coordination. Development of such measures, as well as support for new care delivery models (such as the Patient Centered Medical Home), ${ }^{25}$ have potential to improve the quality of diabetes care for the nearly 6 million people who might have difficulty implementing DSM, or are too old or too vulnerable to be included within the current paradigm.

\section{Acknowledgments}

The authors thank James Passinault for assisting with early development of concept, You-Yu Lee for helping with statistical analyses, and Cathy Emiline-Fegan and Tisha Moore for manuscript preparation and assistance with development of figures and tables.

Supported by NIA-R01 AG021493A and the Ann Arbor VA-GRECC (to C.S.B.). Supported by Ruth L. Kirschstein National Research Service Award from the National Institute on Aging (1F32AG027649-01), the NIHNCRR K12 Mentored Clinical Scholars Program at the University of Michigan, the Ann Arbor VA Geriatric Research, Education and Clinical Center (GRECC), and the John A. Hartford Foundation Center of Excellence in Geriatrics at the University of Michigan (to C.T.C.). Supported by the Johns Hopkins Center for Innovative Medicine Bay-view Scholars and is a Robert Wood Johnson Physician Faculty Scholar (to C.B.). Dr. Wolff had no funding support for the research related to this paper. Supported by a grant from the NIA (R01 AG027010) (K.L.). Supported by a grant from The National Institute on Aging (NIA) provided funding for the Health and Retirement Study (U01 AG09740), which is performed at the Survey Research Center, Institute for Social Research, University of Michigan (D.R.W.). 


\section{REFERENCES}

1. Center for Disease Control. [Accessed April 17, 2009] Diabetes surveillance. 2009. Available at: http://www.cdc.gov/diabetes/statistics/incidence_national.htm

2. Center for Disease Control. [Accessed April 17, 2009] Prevalence of diabetes by age. 2008. Available at: http://www.cdc.gov/diabetes/statistics/prev/national/figbyage.htm

3. UK Prospective Diabetes Study Group. A randomized trial of efficacy of early addition of metformin in sulfonylurea-treated type 2 diabetes. (UKPDS 28). Diabetes Care. 1998; 21:87-92. [PubMed: 9538975]

4. UK Prospective Diabetes Study Group. Tight blood pressure control and risk of macrovascular and microvascular complications in type 2 diabetes: UKPDS 38. BMJ. 1998; 317:703-713. [PubMed: 9732337]

5. UK Prospective Diabetes Study Group. Intensive blood-glucose control with sulphonylureas or insulin compared with conventional treatment and risk of complications in patients with type 2 diabetes (UKPDS 33). Lancet. 1998; 352:837-853. [PubMed: 9742976]

6. UK Prospective Diabetes Study Group. Effect of intensive blood-glucose control with metformin on complications in overweight patients with type 2 diabetes (UKPDS 34). Lancet. 1998; 352:854865. [PubMed: 9742977]

7. Blaum CS, Ofstedal MB, Langa KM, et al. Functional status and health outcomes in older Americans with diabetes mellitus. J Am Geriatr Soc. 2003; 51:745-753. [PubMed: 12757559]

8. Piette JD, Kerr EA. The impact of comorbid chronic conditions on diabetes care. Diabetes Care. 2006; 29:725-731. [PubMed: 16505540]

9. Buse JB, Bigger JT, Byington RP, et al. Action to Control Cardiovascular Risk in Diabetes (ACCORD) trial: design and methods. Am J Cardiol. 2007; 99:21i-33i.

10. Goff DC Jr, Gerstein HC, Ginsberg HN, et al. Prevention of cardiovascular disease in persons with type 2 diabetes mellitus: current knowledge and rationale for the Action to Control Cardiovascular Risk in Diabetes (ACCORD) trial. Am J Cardiol. 2007; 99:4i-20i.

11. ADVANCE Collaborative Group. Effects of a fixed combination of perindopril and indapamide on macrovascular and microvascular outcomes in patients with type 2 diabetes mellitus (the ADVANCE trial): a randomized controlled trial. Lancet. 2007; 370:829-840. [PubMed: 17765963]

12. ADVANCE Collaborative Group. Intensive blood glucose control and vascular outcomes. N Engl J Med. 2008; 358:2560-2572. [PubMed: 18539916]

13. Boyd CM, Darer J, Boult C, et al. Clinical practice guidelines and quality of care for older patients with multiple comorbid diseases: implications for pay for performance. JAMA. 2005; 294:716724. [PubMed: 16091574]

14. Heisler M, Faul JD, Hayward RA, et al. Mechanisms for racial and ethnic disparities in glycemic control in middle-aged and older Americans in the health and retirement study. Arch Intern Med. 2007; 167:1853-1860. [PubMed: 17893306]

15. Heisler M, Smith DM, Hayward RA, et al. Racial disparities in diabetes care processes, outcomes, and treatment intensity. Med Care. 2003; 41:1221-1232. [PubMed: 14583685]

16. Juster FT, Suzman RM. An overview of the health and retirement study. J Human Res. 1995; 30:S7-S56.

17. Blaum CS, Ofstedal MB, Liang J. Low cognitive performance, comorbid disease, and task-specific disability: findings from a nationally representative survey. J Gerontol A Biol Sci Med Sci. 2002; 57:M523-M531. [PubMed: 12145367]

18. Herzog AR, Wallace RB. Measures of cognitive functioning in the AHEAD study. J Gerontol B Psychol Sci Soc Sci. 1997; 52(Spec No):37-48. [PubMed: 9215356]

19. Langa KM, Chernew ME, Kabeto MU, et al. National estimates of the quantity and cost of informal care giving for the elderly with dementia. J Gen Intern Med. 2001; 16:770-778. [PubMed: 11722692]

20. Langa KM, Larson EB, Karlawish JH, et al. Trends in the prevalence and mortality of Alzheimer's disease in the United States. Alzheimers Dement. 2008; 4:1-44. [PubMed: 18631944] 
21. American Diabetes Association. Standards of medical care in diabetes. Diabetes Care. 2008; 31:S12-S54. [PubMed: 18165335]

22. StataCorp. Stata Statistical Software: Release 9. College Station, TX: StataCorp LP; 2005.

23. Brown AF, Mangione CM, Saliba D, et al. Guidelines for improving the care of the older person with diabetes mellitus. J Am Geriatr Soc. 2003; 51 suppl 5:S265-S280. [PubMed: 12694461]

24. Durso SC. Using clinical guidelines designed for older adults with diabetes mellitus and complex health status. JAMA. 2006; 295:1935-1940. [PubMed: 16639053]

25. Association of American medical colleges. The Medical Home. 2008 Available at: http:// www.aamc.org/newsroom/pressrel/2008/medicalhome.pdf. 


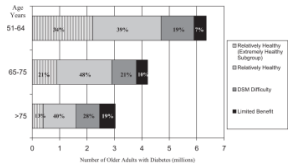

FIGURE 1.

Frequencies of Adults with Diabetes in Clinical Groups by Age. 


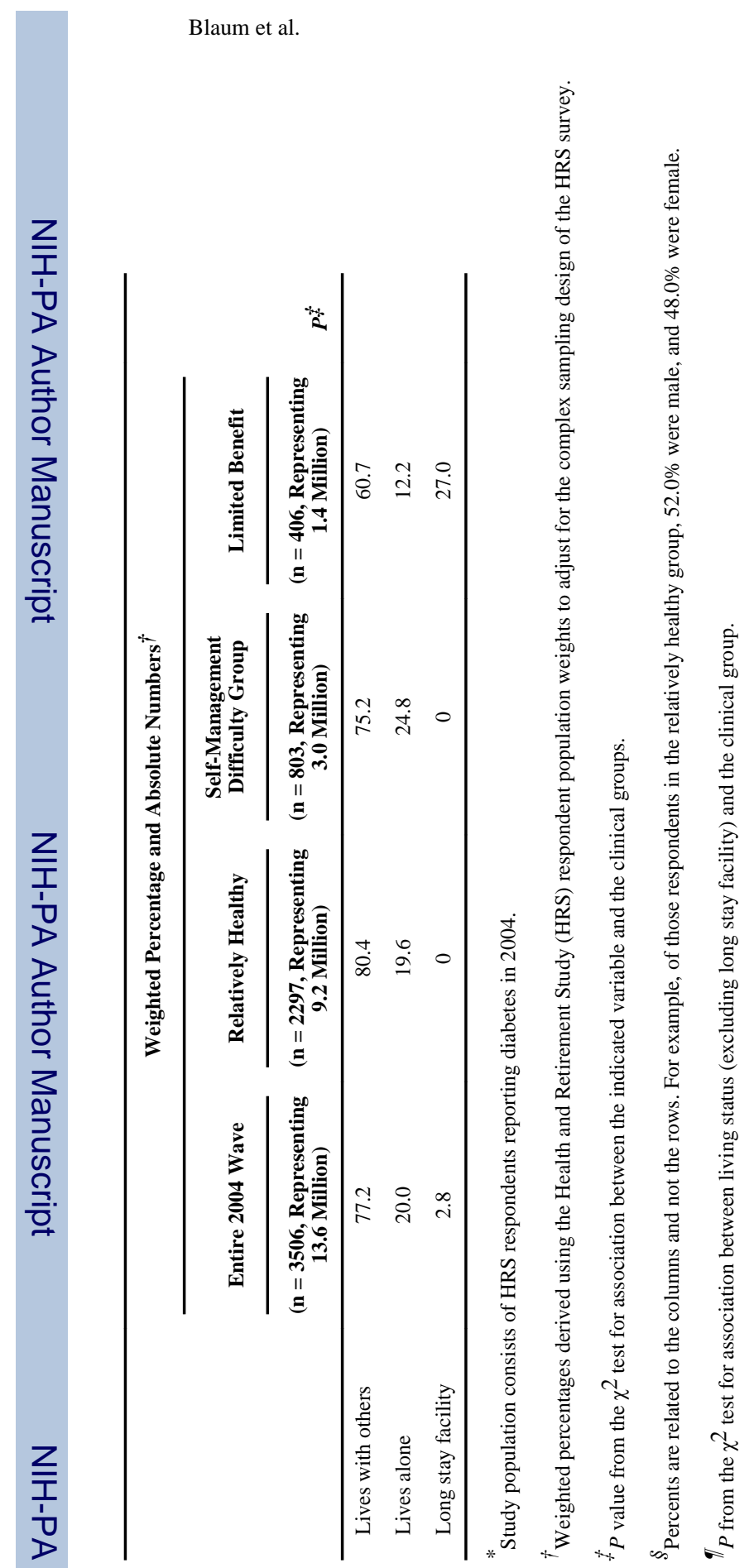

Med Care. Author manuscript; available in PMC 2011 August 9. 
TABLE 2

Diabetes Characteristics of the Clinical Groups*

\begin{tabular}{|c|c|c|c|c|}
\hline & $\begin{array}{c}2004 \text { Respondents } \\
\text { With } \\
\text { HbA }_{1 \mathrm{C}} \text { Values }\end{array}$ & $\begin{array}{c}\text { Relatively Healthy } \\
\text { Group }\end{array}$ & $\begin{array}{c}\text { Diabetes Self-Management } \\
\text { Difficulty Group }\end{array}$ & Limited Benefit Group \\
\hline & $\begin{array}{l}(\mathrm{n}=1132 \\
\text { Representing } \\
\text { 9.9 Million) }\end{array}$ & $\begin{array}{c}(\mathrm{n}=783, \text { Representing } \\
6.5 \text { Million })\end{array}$ & $\begin{array}{c}(\mathrm{n}=260, \text { Representing } \\
\text { 2.4 Million) }\end{array}$ & $\begin{array}{c}(\mathrm{n}=89, \text { Representing } \\
\text { 1.0 Million) }\end{array}$ \\
\hline $\mathrm{HbA}_{1 \mathrm{C}}$ weighted mean ${ }^{\dagger}$ & 7.3 & 7.3 & 7.6 & 7.2 \\
\hline Std. Err & 0.06 & 0.076 & 0.139 & 0.164 \\
\hline Std. Dev & 1.487 & 1.422 & 1.73 & 1.132 \\
\hline$P^{\dagger}$ & & & $P=0.032$ & $P=0.887$ \\
\hline $\mathrm{HbA}_{1 \mathrm{C}}$ weighted median $\S$ & 7 & 6.9 & 7.2 & 7.1 \\
\hline First quartile, third quartile & $6.4,7.9$ & $6.4,7.8$ & $6.5,8.0$ & $6.3,8.0$ \\
\hline Duration $\geq 10 \mathrm{yr}(\%){ }^{q /}$ & 46.4 & 42.3 & 54.0 & 54.8 \\
\hline Std. Err & 0.019 & 0.02 & 0.045 & 0.072 \\
\hline $\mathrm{n}$ & 523 & 330 & 144 & 49 \\
\hline$P$ value $/ l$ & & & $P=0.022$ & $P=0.077$ \\
\hline Insulin $\%$ & 25.4 & 19.5 & 36.9 & 37.1 \\
\hline Std. Err & 0.015 & 0.018 & 0.034 & 0.059 \\
\hline $\mathrm{n}$ & 271 & 145 & 93 & 33 \\
\hline$P^{/ /}$ & & & $P<0.001$ & $P=0.002$ \\
\hline
\end{tabular}

* Subset of 2004 wave respondents with diabetes who had HbA1C values from 2003 Diabetes Study Mail-out Survey. Note that the 2004 wave included ages 51 and older because in that year the cohort was refreshed adding 51-yr-old people. In 2003, the nationally representative ages were 56 and older.

${ }^{\dagger}$ Weighted mean; (linearized standard errors of weighted mean); (standard deviation of weighted mean); $P$.

${ }_{P} P$ values for $\mathrm{HbA}_{1} \mathrm{c}$ variables are for adjusted Wald tests as to whether the mean for the indicated group is significantly different from the mean of the Relatively Healthy Group. For example, the mean $\mathrm{HbA}_{1 \mathrm{c}}$ of $7.6 \%$ among those in the DSM Difficulty Group is significantly different from the $\mathrm{HbA}_{1 \mathrm{c}}$ value of Relatively Healthy Group. We considered displaying $P$ values describing the difference among the variables for all groups but felt the statistical comparison chosen was most relevant.

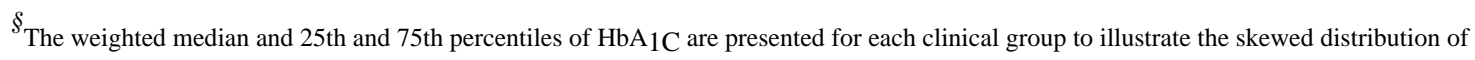
$\mathrm{HbA} 1 \mathrm{C}$. The weighting of medians can only account for response probability, not for survey design, and no statistics were done.

${ }^{I}$ Column proportions; (linearized standard errors of column proportions); number of observations; $P$.

" $P$ for $\%$ variables indicate row association comparisons and are from the Pearson $\chi^{2}$ test for association between the indicated group and the Relatively Healthy Group. For example, in DSM Difficulty Group, the $36.9 \%$ of the people who were taking insulin is a significantly different percent than the percent of the Relatively Healthy Group.

$\mathrm{HbA} 1 \mathrm{C}$ indicates hemoglobin A1C. 
$\mathrm{HbA}_{1 \mathrm{C}}$ indicates hemoglobin $\mathrm{A}_{1 \mathrm{C}}$ 